\title{
Big Bang Tumor Growth and Clonal Evolution
}

\author{
Ruping Sun, ${ }^{1,2}$ Zheng Hu, ${ }^{1,2}$ and Christina Curtis ${ }^{1,2}$ \\ ${ }^{1}$ Departments of Medicine and Genetics, Stanford University School of Medicine, Stanford, California 94305 \\ ${ }^{2}$ Stanford Cancer Institute, Stanford University School of Medicine, Stanford, California 94305 \\ Correspondence: cncurtis@stanford.edu
}

The advent and application of next-generation sequencing (NGS) technologies to tumor genomes has reinvigorated efforts to understand clonal evolution. Although tumor progression has traditionally been viewed as a gradual stepwise process, recent studies suggest that evolutionary rates in tumors can be variable with periods of punctuated mutational bursts and relative stasis. For example, Big Bang dynamics have been reported, wherein after transformation, growth occurs in the absence of stringent selection, consistent with effectively neutral evolution. Although first noted in colorectal tumors, effective neutrality may be relatively common. Additionally, punctuated evolution resulting from mutational bursts and cataclysmic genomic alterations have been described. In this review, we contrast these findings with the conventional gradualist view of clonal evolution and describe potential clinical and therapeutic implications of different evolutionary modes and tempos.

$T^{\text {hen }}$ he multistage model of carcinogenesis developed in the 1950s by Armitage and Doll (Nordling 1953; Armitage and Doll 1954; Fisher 1958) and the clonal evolution theory formalized by Nowell in 1976 (Nowell 1976) have been foundational in shaping our understanding of tumor initiation and progression, as have other early studies (Cairns 1975). Indeed, it has been more than 40 years since Cairns described mutation and natural selection as a driving force in carcinogenesis and proposed that protective mechanisms (including tissue architecture) are in place to limit cancer in multicellular organisms (Cairns 1975). The general importance of these factors is commonly appreciated, although the dynamics of tumor evolution and the resultant patterns of intratumor heterogeneity (ITH) remain poorly understood.
Clonal evolution is an iterative and dynamic process rooted in several key principles. In particular, it assumes that (1) tumors are monoclonal in origin, resulting from the transformation of a single neoplastic cell, as shown based on patterns of cell mosaicism of X-chromosome inactivation (Fialkow 1974); (2) tumor cells continuously accrue somatic aberrations and are subject to ever changing microenvironments, resulting in a heterogeneous population; and (3) tumors progress as a result of strong positive selection for phenotypic traits that promote aggressive behavior and adaptation to diverse microenvironments (Nowell 1976; Greaves and Maley 2012). Although Nowell's description of clonal evolution did not evoke gradualism, wherein the phenotypic traits that characterize tumors develop gradually, this

Editors: Charles Swanton, Alberto Bardelli, Kornelia Polyak, Sohrab Shah, and Trevor A. Graham

Additional Perspectives on Cancer Evolution available at www.perspectivesinmedicine.org

Copyright (C) 2018 Cold Spring Harbor Laboratory Press; all rights reserved; doi: 10.1101/cshperspect.a028381

Cite this article as Cold Spring Harb Perspect Med 2018;8:a028381 
R. Sun et al.

nonetheless became the de facto interpretation, likely influenced by the concept of a multistep process.

Subsequently, Fearon and Vogelstein proposed a schema for colorectal cancer progression, known as the "adenoma-carcinoma sequence," based on the cross-sectional analysis of tumors from different disease stages and the observation that certain driver events were associated with distinct disease stages (Fearon and Vogelstein 1990). In this scenario, tumor initiation is assumed to result from the "stepwise" acquisition of somatic genomic alterations in phenotypically normal or preneoplastic cells accompanied by the "sequential" clonal expansion of cells harboring alterations in "driver" genes that confer a selective growth advantage (Vogelstein et al. 2013). By increasing the fitness (for example, proliferation) of the target cell relative to other premalignant cells, such driver events can result in clonal expansions and selectively sweep through the resident population. Of note, complete selective sweeps resulting in the "fixation" of a clone can only occur provided there is sufficient time before the next driver is acquired. This may be common during the decades often required to initiate a tumor, although premalignant lesions do not necessarily evolve gradually (Maley 2007). Moreover, the linear model has found limited support in recent studies based on high-throughput sequencing of "established" tumors that are sampled after transformation once they become detectable.

Rather, recent studies have revealed patterns consistent with effectively neutral evolution or branched (selective) evolution (Fig. 1). In this review, we discuss paradigm shifts in understanding clonal evolution enabled by the application of next-generation sequencing (NGS) technologies to tumor genomes. For example, we described a Big Bang model of colorectal tumor growth wherein the tumor grows in the absence of stringent selection, consistent with effectively neutral evolution. In this model, the timing of a mutation is the primary determinant of its frequency rather than selection and all major clones persist during growth (Sottoriva et al. 2015). Other studies have corroborated Big Bang dynamics in colorectal
(Sievers et al. 2016a; Uchi et al. 2016; Suzuki et al. 2017) and other tumor types (Ling et al. 2015; Williams et al. 2016), suggesting that effective neutrality may be relatively common. Of note, effectively neutral (or nearly neutral) evolution does not necessarily indicate the absence of selection. Rather, subclones that confer a fitness advantage may be rare or occur late without sufficient time to expand to a detectable frequency (Fig. 1A). In addition, non-cell-autonomous drivers, including stromal factors, and clonal cooperation may contribute to ITH, as suggested by recent studies in patientderived xenografts and genetically engineered mouse models of breast cancer (Cleary et al. 2014; Marusyk et al. 2014). Punctuated evolution resulting from cataclysmic genomic rearrangements and mutational bursts has also been reported in multiple tumor types (Stephens et al. 2011; Lada et al. 2012; Baca et al. 2013; Gao et al. 2016; Notta et al. 2016). Collectively, these data suggest that evolutionary rates can be variable with periods of rapid mutation accumulation, followed by relative stasis. We discuss these models in the broader context of punctuated equilibrium in cancer and contrast this with the conventional gradualist view. Finally, we describe potential clinical and therapeutic implications of different evolutionary modes and tempos.

\section{PRETUMOR PROGRESSION AND FACTORS INFLUENCING CLONAL EXPANSIONS}

The expansion of subclones during preneoplasia depends on several factors, including the fitness benefit that a mutation confers in a phenotypically normal background population. Selectively advantageous mutations in gain-of-function oncogenes and loss-of-function tumor suppressors ultimately contribute to hallmarks seen in all cancers, such as sustained proliferation, invasion, and angiogenesis (Hanahan and Weinberg 2011). These "driver" mutations are often tissue-specific, suggesting context-dependency as in the case of APC mutations in gastrointestinal tumors (Fearon and Vogelstein 1990; Horii et al. 1992; Nakatsuru et al. 1992) and DNMT3 in leukemias (Shlush 

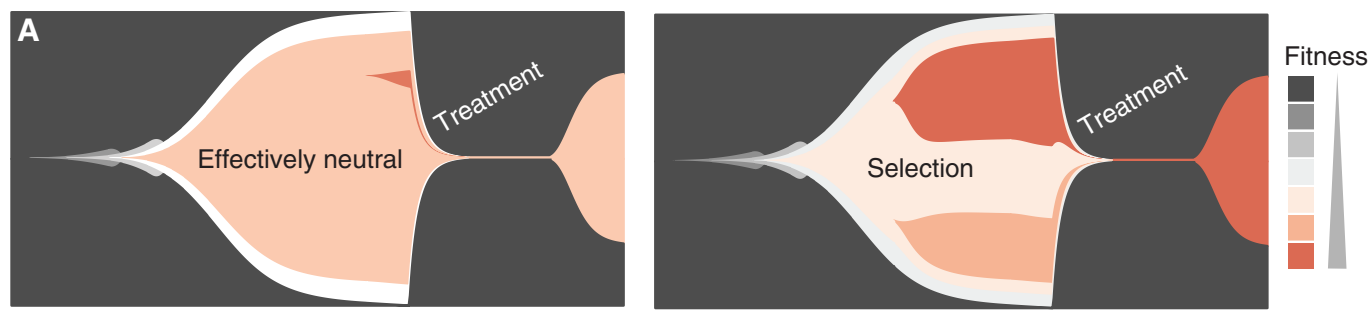
metastasis metastasis
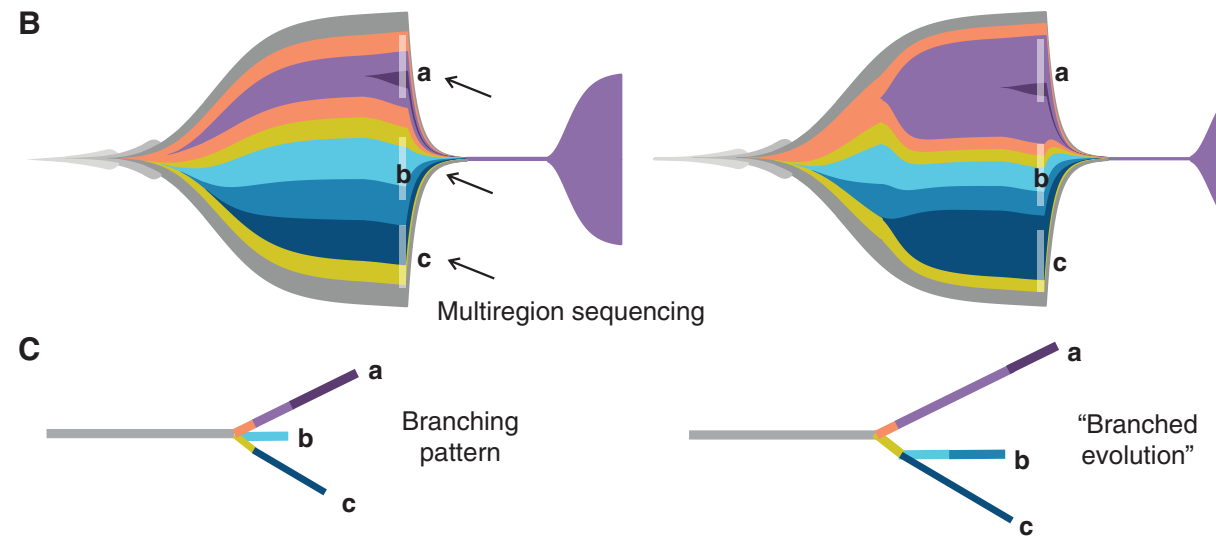

Figure 1. Tumor subclone architecture under different evolutionary models. Muller plots (doi:10.5281/zenodo.240589) illustrate tumor subclone composition under a Big Bang model of effectively neutral growth (left) or a model of stringent selection (right), where descendant genotypes emerge within the parental clone and population size is plotted against time (generations) color coded by $(A)$ clone fitness or $(B)$ clone identity. Multiple stages of tumor progression are depicted beginning with selective sweeps leading up to initiation of the tumor, subsequent growth via effectively neutral evolution or stringent selection, tumor regression in response to treatment, and subsequent relapse or metastasis. In the Big Bang model, all major clones persist in the terminal expansion. As newly arising mutations confer limited fitness benefit, they do not alter subclone composition. Under selection, two late arising selectively advantageous clones outgrow the resident population, resulting in subclonal expansions. Phylogenetic trees constructed from multiregion sampling data (indicated in $B$ ) will (by definition) show branching patterns, irrespective of whether they arise via effectively neutral growth or stringent selection $(C)$. "Branched evolution" refers to the specific scenario of subclone diversification in which multiple selectively advantageous subclones occur in distinct lineages.

et al. 2014). Nonetheless, multiple driver mutations (and clonal expansions) are typically required to initiate a cancer (Vogelstein and Kinzler 2015) and several studies have suggested that the order of mutations influences the phenotype of tumor initiating cells and may be associated with different clinical outcomes (Weaver et al. 2014; Ortmann et al. 2015; see also Kent and Green 2017).

The lower the fitness advantage conferred by a mutation in a given cell generation, the higher the population diversity and lower the chance of fixation in an exponentially growing population (Durrett et al. 2011). If individual cells acquire mutations that confer similar fitness advantages at similar times, multiple lineages can coexist, potentially contributing to clonal interference. Of note, clonal interference is likely to be common in large asexual populations and to impede the fixation of subclones (Fogle et al. 2008). Moreover, the efficacy of selection is proportional to the effective population size (Charlesworth 2009), such that smaller effective population sizes can be more strongly influenced by genetic drift, as discussed in detail elsewhere (Hu et al. 2017; Sottoriva 
R. Sun et al.

et al. 2017). Because premalignant populations ( prior to transformation) are presumably small, such neutral mutations may more readily become fixed due to random genetic drift.

As noted by Cairns (1975), tissue architecture is one protective mechanism that can limit cancer development. Indeed, Martens et al. (2011) reported that spatial structure minimizes selection and subclonal expansions, thereby delaying the waiting time to cancer. Additionally, experiments that measured the competitive fitness advantage of common driver mutations in stem cells during tumor initiation in the mouse small intestine revealed that mutation fixation is highly inefficient due to hierarchical tissue architecture and stochastic stem cell replacement (Vermeulen et al. 2013). This was despite the two- to fourfold selective advantage (probability of fixation in the crypt) of APC or KRAS mutant relative to wild-type stem cells. Thus, tissue niches that harbor tumor-initiating cells, such as the intestinal crypt, can restrict clonal expansions. More generally, subclone expansions can be influenced by the fitness benefit that a selectively advantageous mutation confers relative to the background population, tissue spatial structure, the effective population size, deleterious passenger events, and clonal interference.

It has long been assumed that the cell of origin in many cancers is a self-renewing stem cell and that this is the functional unit on which selection operates (Greaves and Maley 2012). In this scenario, cancer reflects a state in which the normal balance of self-renewal and differentiation is lost. Due to the limited proliferative potential of nonstem cells, only mutations in stem cells result in clonal expansions within the niche (Sprouffske et al. 2013), and this contributes to a decreased effective population size. The observation that higher stem cell activity is associated with poor prognosis lends support to this model (Greaves 2015; Ng et al. 2016). A recent study used lineage tracing in mice to evaluate the variability in cancer risk across different organs and found that this is dictated by stem cell mutagenesis and extrinsic factors that influence the proliferation of these long-lived populations (Zhu et al. 2016). Other studies have reported an association between per tissue cancer risk and the lifetime number of stem cell divisions (Tomasetti and Vogelstein 2015), although the relative contribution of total stem cell divisions, environment, and heritability has been debated (Noble et al. 2015; Rozhok et al. 2015).

The above factors contribute to the high degree of detectable ITH or genetic diversity noted in preinvasive lesions, including colorectal adenomas (Kang et al. 2015; Sottoriva et al. 2015; Sievers et al. 2016b; Uchi et al. 2016), ductal carcinoma in situ (DCIS) (Allred et al. 2008), and Barrett's esophagus (BE) (Weaver et al. 2014) at the mutational and copy-number levels. Indeed, Boveri proposed that chromosomal instability (CIN), resulting from whole or partial chromosomal gains and losses, was a critical event in tumorigenesis more than a century ago (Boveri and Boveri 1929). CIN may occur relatively early based on its prevalence in premalignancy (Rabinovitch et al. 1989; Heaphy et al. 2009; Cucco et al. 2014). For example, genomic instability was shown to increase in atypical ductal hyperplasia $(\mathrm{ADH})$ relative to normal tissue but to plateau in DCIS to invasive disease, suggesting that invasive carcinomas evolve from or in parallel to DCIS (Heaphy et al. 2009). This is in-line with more recent studies indicating that preinvasive breast tumors are characterized by lineage heterogeneity both within and between lesions (Weng et al. 2015) and that copy-number states are relatively static during breast tumor progression (Gao et al. 2016). Intriguingly, a comparison of $\mathrm{BE}$ and adjacent esophageal adenocarcinoma (ESCA) revealed significant heterogeneity in the spectrum of somatic alterations with surprisingly little overlap. These findings may reflect early divergence in the lineage that formed the carcinoma and ongoing evolution or potentially the evolution of multiple parallel lineages.

To date, most efforts have focused on sequencing established tumors, wherein the timing of genomic alterations leading to up to the last clonal expansion can be obscured because mutations that accrue in the initiating tumor cell will be clonal (public) and present in all cells of the final tumor. Efforts to characterize somatic alterations present in phenotypically 
Big Bang Tumor Growth and Clonal Evolution

normal cells and preinvasive lesions are therefore of critical importance for understanding the earliest events in tumorigenesis and may pave the way for earlier detection and prevention. For example, clonal hematopoiesis is relatively common, occurring in $10 \%$ of individuals over age 65 and is a strong risk factor for subsequent hematologic cancer (Genovese et al. 2014). Similar studies are more challenging to undertake in normal tissues given the need to biopsy, although this has been performed on a limited basis with accessible epithelial sites (Martincorena et al. 2016). More generally, longitudinal specimens can provide unique insights into evolutionary dynamics, but practical and ethical considerations limit their routine collection. Fortunately, lineage tracing techniques continue to advance and represent powerful tools to identify the cell of origin, functional drivers, and temporal ordering of tumor initiating events in experimental systems.

\section{POSTTRANSFORMATION CLONAL EVOLUTION}

Under the de facto model of clonal evolution, both tumor initiation and subsequent growth after transformation proceed in a "linear" sequential fashion in which successively more fit clones selectively sweep through the resident population (Greaves and Maley 2012; Yates and Campbell 2012). The advent of NGS has enabled the characterization of ITH at the (epi)genomic and transcriptomic levels at unprecedented resolution in diverse solid tumors (Gerlinger et al 2012, 2014; Bashashati et al. 2013; Sottoriva et al. 2013a,b, 2015; de Bruin et al. 2014; Zhang et al. 2014; Kim et al. 2015a,b; McPherson et al. 2016). Importantly, patterns of genetic ITH encode the tumor's evolutionary history by surreptitiously recording the errors made during cell division. Given the significant spatial constraints in solid tumors contributing to sampling bias, such patterns are more readily captured using multiregion sequencing (MRS) and/or single cell sequencing than with a single bulk tumor sample. Several studies have revealed private putative driver events on the branches of phylogenetic trees, indicative of the presence of multiple selectively advantageous subclones, whereas public or clonal events are on the trunk of the tree (Fig. 1) (Gerlinger et al. 2012, 2014; Bashashati et al. 2013; de Bruin et al. 2014; Zhang et al. 2014; Kim et al. 2015a; Weng et al. 2015; McPherson et al. 2016). This has been referred to as "branched evolution" in contrast to the linear clonal succession model, in which a selectively advantageous mutation has time to sweep through the population before the acquisition of additional drivers (Greaves 2015). These and other studies point to the lack of support for linear evolution in most established human cancers (Davis et al. 2017; Hu et al. 2017).

High levels of ITH at the genomic, transcriptomic, cellular, and phenotypic levels have been reported in diverse tumor types and pose challenges for precision medicine with implications for patient stratification and the efficacy of molecularly targeted therapies. For example, it is presently not known whether subclonal driver mutations are suboptimal therapeutic targets or the extent to which genomic heterogeneity is functional. Likewise, if different regions of a patient's tumor are classified into multiple molecular subgroups, as observed in glioblastoma (Sottoriva et al. 2013a), this could complicate treatment decision making.

Of note, phylogenies are by definition branching diagrams, and so-called "branched" phylogenies do not imply branched clonal evolution (Fig. 1). Other scenarios, including effectively neutral evolution have been reported (Ling et al. 2015; Sottoriva et al. 2015; Uchi et al. 2016), as discussed below. In rapidly growing spatially structured populations, there is a high chance of detecting genetic divergence between regionally segregated samples due to enhanced genetic drift. Therefore, methods to systematically evaluate whether observed patterns of ITH are attributable to ongoing selection or can be explained by neutral drift are needed.

\section{BIG BANG TUMOR GROWTH DYNAMICS}

Mounting evidence suggests that, at least in some tumors, selection may not be readily detectable after initiation. In particular, we recent- 
R. Sun et al.

ly described a Big Bang model of colorectal tumor growth, wherein after transformation, some colorectal tumors grow as a single terminal expansion in the absence of stringent selection, compatible with effectively neutral evolution (Sottoriva et al. 2015). Hence, the constellation of driver alterations necessary for tumor initiation are apparently sufficient for subsequent expansion, such that further selection is minimal. In the Big Bang model, the timing of a mutation dictates its cellular prevalence in the final tumor. As such, most detectable subclonal (private) alterations occur early during tumor expansion, whereas late-arising mutations will be undetectable as they are diluted by population growth. Thus, subclone composition remains relatively stable with major clones persisting during growth (Fig. 2).

The Big Bang model generates a number of testable predictions, several of which were examined through detailed sampling of distant tumor regions, single gland, and single cell profiling at the mutational and copy-number levels. These data revealed uniformly high ITH at multiple spatial scales. Moreover, glands from distant tumor regions were found to have similar

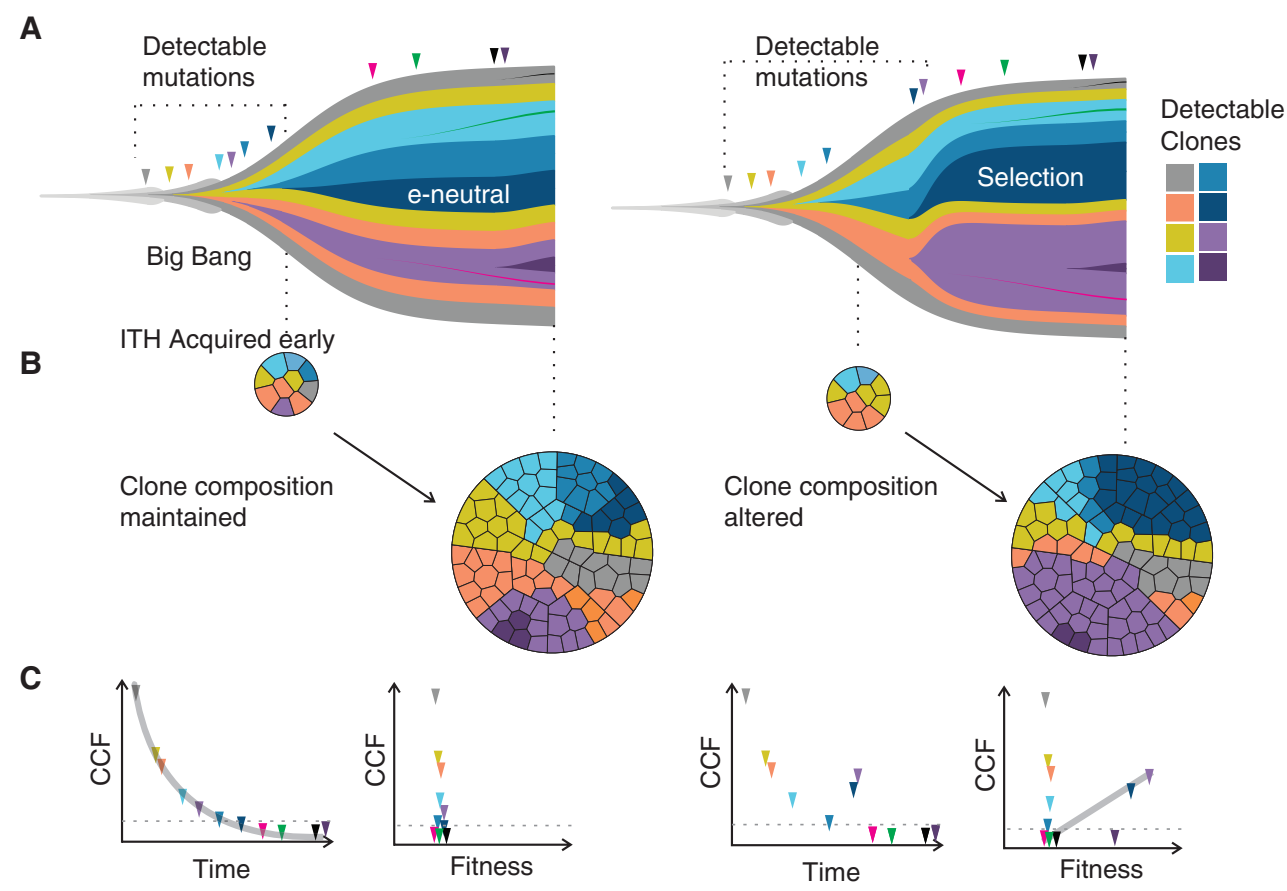

Figure 2. Comparison of the Big Bang versus branched evolution models. (A) Muller plots illustrate tumor subclone composition under a Big Bang model of effectively neutral growth (e-neutral, left) or a model of stringent selection (right), where descendant genotypes emerge within the parental clone and population size is plotted against time (generations). Under the Big Bang model, only early mutations can be detected via bulk sampling. In contrast, under selection late arising mutations that confer strong fitness benefit can attain detectable frequencies. (B) In the Big Bang model, most detectable intratumor heterogeneity (ITH) (due to private alterations) is acquired early and subclone composition is preserved over time. In contrast, under stringent selection, subclone composition varies with the increase and decrease of clones. $(C)$ In the Big Bang model, the timing of a mutation is the major determinant of its cellular prevalence or cancer cell fraction (CCF), with late arising mutations present at increasingly low-frequency to the point that they decrease below detection limits. Although mutations continuously accrue, they are selectively neutral and do not confer significant fitness benefits to the existing tumor cell population. Under stringent selection, tumor growth similarly dilutes mutational frequency. However, clones that confer a selective growth advantage relative to the background population show outgrowth, such that increases in CCF correlate with fitness. 
mitotic ages suggesting that they derive from the same clonal expansion, as in prior studies (Siegmund et al. 2011; Sottoriva et al. 2013b). Additionally, mutations within a gland were found to be clonal despite being private in the bulk tumor, suggesting relatively old clonal expansions wherein mutations had time to be fixed or lost due to neutral drift.

Such dense sampling and single gland profiling also revealed fresh insights into subclone architecture that would be obscured by bulk profiling. In particular, patterns of genetic variegation or subclone mixing were observed such that single glands from distant ( $>3 \mathrm{~cm}$ apart) tumor regions were found to harbor identical subclonal somatic single nucleotide variants (sSNV) or copy-number alteration (CNA) breakpoints (Sottoriva et al. 2015). Although this could potentially be explained by departures from an infinite sites model, wherein each site in the genome is mutated only once, this seems unlikely given the frequent patterns of mutational sharing even amongst passenger alterations. Whole gland migration could also potentially explain the presence of the same mutation in spatially separated tumor regions, but is unlikely in a large tumor due to spatial constraints. As another explanation, it was proposed that in a small early primary tumor, clones with abnormal mobility could mix efficiently and subsequently be "scattered" to distant tumor regions during growth. This is compatible with the finding that most detectable ITH occurs very early, before the lesion is clinically detectable. Alternatively, it is plausible that these patterns arise from repeatedly sampling large clone boundaries. Of note, only extremely early subclonal mutations are likely to show subclone mixing, whereas private mutations tend to be restricted to one tumor region due to spatial constraints. In a recent study, Suzuki et al. (2017) reported further evidence for subclone mixing in colorectal cancers by defining both ubiquitous mutations present in all tumor sectors and those that were subclonal in more than one sector based on deep targeted sequencing. Such patterns may not be unique to the colon, as subclone mixing has also been reported in breast cancers (Yates et al. 2015).
Big Bang Tumor Growth and Clonal Evolution

By simulating spatial tumor growth and performing inference on the genomic data, we further verified that most detectable ITH indeed occurred early while the tumor was on the order of $10^{4}-10^{5}$ cells, long before it was clinically evident (Sottoriva et al. 2015). Using this computational framework to infer patient-specific tumor dynamics indicated that subclone fitness differences were present in most colorectal carcinomas. However, taken together with the genomic data, these results suggested that although selection was likely operative, it often left a weak signal on the tumor genome. This is not altogether surprising because numerous factors can impede the efficacy of selection, including tumor growth itself. However, these findings also highlight the need for systematic approaches to quantify the levels and impact of natural selection on cancer genomes.

Several other MRS studies have corroborated Big Bang growth dynamics in colorectal (Bozic et al. 2016; Sievers et al. 2016a; Uchi et al. 2016; Suzuki et al. 2017) and other tumor types (Ling et al. 2015; Williams et al. 2016). For example, Uchi et al. (2016) performed bulk MRS and similarly suggested that effectively neutral growth could potentially explain the observed patterns of ITH. In another study (Ling et al. 2015), MRS coupled with dense genotyping in a hepatocellular tumor revealed extremely high ITH. By examining the distribution of variant allele frequencies, the investigators report failure to reject a "null" neutral model assuming exponential growth of a wellmixed population. These studies differ in experimental design and approach, but suggest that Big Bang tumor growth and effective neutrality may be relatively common.

\section{PUNCTUATED MUTATIONAL PROCESSES AND CLONAL STASIS}

In the Big Bang tumor growth model, once the precursor lineage has acquired the requisite driver mutations (potentially gradually), the tumor is initiated (in a punctate manner) and grows as a single expansion in a neutral fashion. This implies that the initiated tumor has achieved a fitness plateau in which further mu- 
R. Sun et al.

tations do not confer a selective growth advantage, potentially corresponding to a period of evolutionary ( phenotypic) stasis.

Further evidence for punctuated mutational processes and periods of stasis have been noted in other cancers at the level of sSNVs and indels, as well as CNAs and large scale structural variants (SVs) (Lawrence et al. 2013; Wang et al. 2014; Gao et al. 2016). For example, single nuclei sequencing of triple-negative breast cancers similarly showed that CNAs occur early during tumor evolution and that intermediate clones with gradual gain or loss of genomic segments were seldom observed during transitions to aneuploidy (Gao et al. 2016). Similar to the Big Bang model, these data suggest that early bursts of CNAs are followed by stable clonal expansions that form the final tumor, and support prior assertions that the linear paradigm may not apply to breast cancer (Polyak 2008). Additionally, catastrophic events such as chromothrypsis have been reported in several tumor types, in which oscillating copy-number states occur in adjacent regions, restricted to one or a few chromosomes due to surges of chromosomal rearrangements that occur in a single or few cell cycles (Stephens et al. 2011; Rausch et al. 2012; Notta et al. 2016) or during micronuclei formation (Zhang et al. 2015). Such events are associated with mitotic errors and the simultaneous mutation of multiple drivers, resulting in extensive ITH in short time scales. In particular, in pancreatic tumors, chromothripsis is thought to be a common initiating event with two-thirds of cases showing evolutionary trajectories consistent with punctuated equilibrium (Notta et al. 2016). As a result of mutational bursts, tumor cells may attain greater fitness than would be possible through gradual alterations, owing to the acquisition of multiple driver alterations (Korbel and Campbell 2013). Chromoplexy is another catastrophic event characterized by chains of translocations resulting from punctuated bursts of interchromosomal SVs and CNAs (Baca et al. 2013). Additional mutational processes include localized hypermutation involving small-scale alterations (sSNVs and indels) as in the case of kataegis, which is attributable to cytosine deam- ination catalyzed by AID/APOBEC proteins (Lada et al. 2012; D'Antonio et al. 2016). Impaired DNA mismatch repair (MMR) leading to microsatellite instability can also increase the rate of sSNVs and indels. Stochastic karyotypic changes due to aneuploidy and SVs have also been noted to promote cancer progression in a discontinuous fashion (Heng et al. 2006).

These and other data strongly suggest that mutation rates are not constant during tumor progression. Moreover, telomere crisis has been reported to cause elevated genomic instability during early tumor progression, which then decreases once telomerase function is restored (Chin et al. 2004). Additionally, simulation studies suggest that deleterious mutations associated with increased mutation rates can, in turn, impede the efficiency of positive selection (McFarland et al. 2013). Thus, cellular fitness tradeoffs are associated with elevated mutational levels, a concept known as Muller's ratchet (Lynch et al. 1993). A related phenomenon in which deleterious alterations drive population extinction, termed mutational meltdown, has also been proposed and could contribute to changes in evolutionary tempo. Collectively, these findings suggest that the linear, gradualist model does not accurately describe clonal evolution in many human cancers.

\section{SIGNALS OF SELECTION IN CANCER GENOMES}

A focus of current research is to delineate the levels of selection are operative at different stages of progression in distinct tumor types and the extent to which it leaves a detectable signal on the genome. In some tumors, signals of selection are evident, as in the case of the punctuated mutational processes described above or convergent evolution. As an example of the latter, in renal cell carcinoma (RCC), multiple alterations in the same gene or pathway were noted in different tumor regions, consistent with selection of the same functional consequence via convergent evolution (Gerlinger et al 2012; Barber et al. 2015). To date, reports of convergent genomic evolution have been relatively few, but this may be more apparent at the pheno- 
Big Bang Tumor Growth and Clonal Evolution

typic level. The more general scenario of branched evolution in which multiple putative subclonal driver events occur on distinct branches of a phylogenetic tree is also suggestive of selection, although in many cases the functional effect of a putative driver in a given context is not known. Mutational heterogeneity (Lawrence et al. 2013) can also complicate the interpretation of such patterns. Of note, these lines of evidence are qualitative rather than quantitative.

Importantly, tumors growing under stringent selection (e.g., branched evolution) versus "effective" neutrality are expected to yield distinct patterns of ITH that can be quantitatively evaluated through NGS. Strong positive selection is assumed to be common in tumor samples and expected to alter tumor subclonal composition (Gerlinger et al. 2012, 2014; NikZainal et al. 2012; de Bruin et al. 2014; Wang et al. 2014; Yates et al. 2015). In particular, under stringent selection, late-arising driver mutations accompanied by hitchhiking passengers in the same lineage can attain high-frequency and manifest as "subclonal clusters" in bulk tumor sequencing data. In contrast, Big Bang tumor growth implies effectively neutral evolution in which mutations that were selected for are truncal and subsequent mutations are passenger events. In this model, timing the fundamental determinant of its frequency and most detectable ITH occurs early. As such, all major clones persist and subclonal composition is preserved (Fig. 2).

Attempts to distinguish modes of tumor evolution should account for tumor spatial structure and sampling bias as this can profoundly impact clonal dynamics, as discussed in previous sections. Additionally, from a practical perspective, single sample bulk sequencing may fail to detect selection when private mutations become regionally fixed and hence appear public. Moreover, subclonal diversification itself does not necessarily indicate ongoing selection, as neutral drift can be significant in spatially structured populations resulting in high-frequency region-specific mutations.

As knowledge of tumor growth dynamics may inform more effective treatment strategies, there remains a crucial need to quantitatively assess this in a patient-specific manner. To this end, quantitative comparisons of between region ITH in clinical samples and comparison to values obtained in tumors simulated under different evolutionary modes may help to resolve these patterns, as was recently reported (Sun et al. 2017).

\section{CLINICAL IMPLICATIONS OF PUNCTUATED EVOLUTION AND BIG BANG DYNAMICS}

A tumor's evolutionary trajectory may dictate its future course. As such, understanding the mode and tempo of tumor evolution may inform whether it is more likely to progress to invasion, to metastasize or be intrinsically resistant to therapy. It is therefore plausible that ITH represents a general prognostic biomarker. Indeed, in a seminal early study, Maley et al. (2006) reported that clonal diversity predicts progression from BE to ESCA. Subsequent efforts have shown an association between ITH and poor outcome in established tumors (Mroz and Rocco 2013; Mroz et al. 2013). Further studies should help to resolve which molecular features and measures of ITH are most predictive in specific tumor types and disease stages.

In a similar vein, we proposed that some tumors characterized by Big Bang dynamics may be "born to be bad," wherein malignant potential is specified by the public driver alterations present in the initiating tumor cell (Sottoriva et al. 2015). Effectively neutral evolution can also confound screening efforts because apparently stable tumors may not progress further. As such, it will be important to distinguish between these scenarios and to determine whether tumors characterized by effectively neutral evolution versus stringent selection have different clinical outcomes. Furthermore, effectively neutral growth results in high levels of genetic diversity, thereby increasing the likelihood that preexisting variants will be intrinsically resistant to therapy. Indeed, the landscape of rare and largely undetectable subclones potentially provides a rich substrate for resistance in the context of treatment selective pressure. 
R. Sun et al.

The shift away from a linear gradualist view toward punctuated evolution also has clinical ramifications. Traditionally, the linear model implied that aggressive cancers, including those of the pancreas, evolve gradually and only become invasive and detectable late during the evolutionary course (Yachida et al. 2010). However, current data suggest that early cataclysmic events initiate many such tumors, with attendant implications for the early specification of malignant potential.

The relationship between mutational burden and clinical outcome has been studied for some time. For example, colorectal cancers with MMR deficiency resulting in hypermutation are associated with favorable prognosis relative to microsatellite stable (MSS) tumors (Sinicrope and Yang 2011). High mutation load also contributes to an elevated number of tumorspecific immunogenic neoantigens, which in turn, confers increased sensitivity to immunecheckpoint blockage in MMR-deficient colorectal tumors (Le et al. 2015) and in melanomas (Snyder et al. 2014). In contrast, aneuploidy is associated with markers of immune evasion and poor response to immunotherapy (Davoli et al. 2017). More recently, the impact of neoantigen ITH on anti-tumor immunity and response to immunotherapy has been investigated, suggesting a delicate balancing act (McGranahan et al. 2016). In light of torrent of immunotherapybased clinical trials, it remains essential to develop improved strategies for patient stratification that account for the many factors that can influence immunogenicity (Giannakis et al. 2016).

\section{DISCUSSION}

Recent cancer genome sequencing efforts coupled with computational modeling have revealed abundant evidence for neutral and punctuated evolutionary processes, which are inadequately explained by the conventional gradualist linear model. These studies point to a revised model of clonal evolution based on "punctuated equilibrium" in which episodes of gradual mutation accumulation are interrupted by punctate mutational bursts and inter- spersed with long periods of clonal stasis (Cross et al. 2016). Within this view, it is important to consider the distinct stages of tumor evolution in concert, including pretumor progression, initiation, subsequent growth, and in some cases, metastatic spread. The fact that much of tumor progression is occult and obscured by the final clonal expansion(s) and that time scales are relative has potentially contributed to confusion regarding evolutionary tempos.

A punctuated equilibrium model of cancer also better explains numerous clinical observations, including the high frequency of interval cancers detected between screenings, the higher prevalence of premalignant and noninvasive tumors relative to invasive cancers, and the inability to predict disease progression, as would be anticipated under a linear model. More cancers may be found to show punctuated mutational processes when all forms of somatic alterations are robustly accounted for since exome sequencing is inadequate for detecting SVs and single cell assays are prone to noise. Beyond this, there remains a need to delineate the genotype to phenotype map in cancer because selection operates on phenotypes and changes in clone composition may not be functionally relevant. Ideally, such efforts will be coupled with longitudinal sampling toward a better understanding of evolutionary modes and tempos.

\section{ACKNOWLEDGMENTS}

This work was funded by awards from the National Institutes of Health (NIH) (R01CA182514), Susan G. Komen Foundation (IIR13260750), and the Breast Cancer Research Foundation (BCRF-16-032) to C.C. Z.H. is supported by an Innovative Genomics Initiative (IGI) Postdoctoral Fellowship.

\section{REFERENCES}

${ }^{*}$ Reference is also in this subject collection.

Allred DC, Wu Y, Mao S, Nagtegaal ID, Lee S, Perou CM, Mohsin SK, O'Connell P, Tsimelzon A, Medina D. 2008. Ductal carcinoma in situ and the emergence of diversity during breast cancer evolution. Clin Cancer Res 14: 370 378 . 
Big Bang Tumor Growth and Clonal Evolution

Armitage P, Doll R. 1954. The age distribution of cancer and a multi-stage theory of carcinogenesis. Br J Cancer 8: $1-12$.

Baca SC, Prandi D, Lawrence MS, Mosquera JM, Romanel A, Drier Y, Park K, Kitabayashi N, MacDonald TY, Ghandi M, et al. 2013. Punctuated evolution of prostate cancer genomes. Cell 153: 666-677.

Barber LJ, Davies MN, Gerlinger M. 2015. Dissecting cancer evolution at the macro-heterogeneity and micro-heterogeneity scale. Curr Opin Genet Dev 30: 1-6.

Bashashati A, Ha G, Tone A, Ding J, Prentice LM, Roth A, Rosner J, Shumansky K, Kalloger S, Senz J, et al. 2013. Distinct evolutionary trajectories of primary high-grade serous ovarian cancers revealed through spatial mutational profiling. J Pathol 231: 21-34.

Boveri T, Boveri M. 1929. The origin of malignant tumors, ix, 119 p. Williams \& Wilkins, Baltimore, MD.

Bozic I, Gerold JM, Nowak MA. 2016. Quantifying clonal and subclonal passenger mutations in cancer evolution. PLoS Comput Biol 12: e1004731.

Cairns J. 1975. Mutation selection and the natural history of cancer. Nature 255: 197-200.

Charlesworth B. 2009. Fundamental concepts in genetics: Effective population size and patterns of molecular evolution and variation. Nat Rev Genet 10: 195-205.

Chin K, de Solorzano CO, Knowles D, Jones A, Chou W, Rodriguez EG, Kuo WL, Ljung BM, Chew K, Myambo K, et al. 2004. In situ analyses of genome instability in breast cancer. Nat Genet 36: 984-988.

Cleary AS, Leonard TL, Gestl SA, Gunther EJ. 2014. Tumour cell heterogeneity maintained by cooperating subclones in Wnt-driven mammary cancers. Nature 508: 113-117.

Cross W, Graham TA, Wright NA. 2016. New paradigms in clonal evolution: Punctuated equilibrium in cancer. J Pathol 240: 126-36.

Cucco F, Servadio A, Gatti V, Bianchi P, Mannini L, Prodosmo A, De Vitis E, Basso G, Friuli A, Laghi L, et al. 2014. Mutant cohesin drives chromosomal instability in early colorectal adenomas. Hum Mol Genet 23: 6773-6778.

D’Antonio M, Tamayo P, Mesirov JP, Frazer KA. 2016. Kataegis xepression signature in breast cancer is associated with late onset, better prognosis, and higher HER2 levels. Cell Rep 16: 672-683.

Davis A, Gao R, Navin N. 2017. Tumor evolution: Linear, branching, neutral or punctuated? Biochim Biophys Acta doi: 10.1016/j.bbcan.2017.01.003.

Davoli T, Uno H, Wooten EC, Elledge SJ. 2017. Tumor aneuploidy correlates with markers of immune evasion and with reduced response to immunotherapy. Science 355: eaaf8399.

de Bruin EC, McGranahan N, Mitter R, Salm M, Wedge DC, Yates L, Jamal-Hanjani M, Shafi S, Murugaesu N, Rowan AJ, et al. 2014. Spatial and temporal diversity in genomic instability processes defines lung cancer evolution. Science 346: 251-256.

Durrett R, Foo J, Leder K, Mayberry J, Michor F. 2011. Intratumor heterogeneity in evolutionary models of tumor progression. Genetics 188: 461-477.

Fearon ER, Vogelstein B. 1990. A genetic model for colorectal tumorigenesis. Cell 61: 759-767.
Fialkow PJ. 1974. Human genetic markers as tracers of tumour histogenesis. J Clin Pathol Suppl (R Coll Pathol) 7: $11-15$.

Fisher JC. 1958. Multiple-mutation theory of carcinogenesis. Nature 181: 651-652.

Fogle CA, Nagle JL, Desai MM. 2008. Clonal interference, multiple mutations and adaptation in large asexual populations. Genetics 180: 2163-2173.

Gao R, Davis A, McDonald TO, Sei E, Shi X, Wang Y, Tsai PC, Casasent A, Waters J, Zhang H, et al. 2016. Punctuated copy number evolution and clonal stasis in triplenegative breast cancer. Nat Genet 48: 1119-1130.

Genovese G, Kähler AK, Handsaker RE, Lindberg J, Rose SA, Bakhoum SF, Chambert K, Mick E, Neale BM, Fromer M, et al. 2014. Clonal hematopoiesis and blood-cancer risk inferred from blood DNA sequence. N Engl J Med 371: 2477-2487.

Gerlinger M, Gerlinger M, Rowan AJ, Horswell S, Larkin J, Endesfelder D, Gronroos E, Martinez P, Matthews N, Stewart A, Tarpey P, et al.2012. Intratumor heterogeneity and branched evolution revealed by multiregion sequencing. N Engl J Med 366: 883-892.

Gerlinger M, Horswell S, Larkin J, Rowan AJ, Salm MP, Varela I, Fisher R, McGranahan N, Matthews N, Santos CR, et al. 2014. Genomic architecture and evolution of clear cell renal cell carcinomas defined by multiregion sequencing. Nat Genet 46: 225-233.

Giannakis M, Mu XJ, Shukla SA, Qian ZR, Cohen O, Nishihara R, Bahl S, Cao Y, Amin-Mansour A, Yamauchi M, et al. 2016. Genomic correlates of immune-cell infiltrates in colorectal carcinoma. Cell Rep 17: 1206.

Greaves M. 2015. Evolutionary determinants of cancer. Cancer Discov 5: 806-820.

Greaves M, Maley CC. 2012. Clonal evolution in cancer. Nature 481: 306-313.

Hanahan D, Weinberg RA. 2011. Hallmarks of cancer: The next generation. Cell 144: 646-674.

Heaphy CM, Bisoffi M, Joste NE, Baumgartner KB, Baumgartner RN, Griffith JK. 2009. Genomic instability demonstrates similarity between DCIS and invasive carcinomas. Breast Cancer Res Treat 117: 17-24.

Heng HH, Stevens JB, Liu G, Bremer SW, Ye KJ, Reddy PV, Wu GS, Wang YA, Tainsky MA, Ye CJ. 2006. Stochastic cancer progression driven by non-clonal chromosome aberrations. J Cell Physiol 208: 461-472.

Horii A, Nakatsuru S, Miyoshi Y, Ichii S, Nagase H, Ando H, Yanagisawa A, Tsuchiya E, Kato Y, Nakamura Y. 1992. Frequent somatic mutations of the APC gene in human pancreatic cancer. Cancer Res 52: 6696-6698.

Hu Z, Sun R, Curtis C. 2017. A population genetics perspective on the determinants of intra-tumor heterogeneity. Biochim Biophys Acta 1867: 109-126.

Kang H, Salomon MP, Sottoriva A, Zhao J, Toy M, Press MF, Curtis C, Marjoram P, Siegmund K, Shibata D. 2015. Many private mutations originate from the first few divisions of a human colorectal adenoma. J Pathol 237: $355-362$.

* Kent DG, Green AR. 2017. Order matters: The order of somatic mutations influences cancer evolution. Cold Spring Harb Perspect Med doi: 10.1101/cshperspect.a027060. 
R. Sun et al.

Kim H, Zheng S, Amini SS, Virk SM, Mikkelsen T, Brat DJ, Grimsby J, Sougnez C, Muller F, Hu J, et al. 2015a. Wholegenome and multisector exome sequencing of primary and post-treatment glioblastoma reveals patterns of tumor evolution. Genome Res 25: 316-327.

Kim TM, Jung SH, An CH, Lee SH, Baek IP, Kim MS, Park SW, Rhee JK, Lee SH, Chung YJ. 2015b. Subclonal genomic architectures of primary and metastatic colorectal cancer based on intratumoral genetic heterogeneity. Clin Cancer Res 21: 4461-4472.

Korbel JO, Campbell PJ. 2013. Criteria for inference of chromothripsis in cancer genomes. Cell 152: 1226-1236.

Lada AG, Dhar A, Boissy RJ, Hirano M, Rubel AA, Rogozin IB, Pavlov YI. 2012. AID/APOBEC cytosine deaminase induces genome-wide kataegis. Biol Direct 7: 47; discussion 47.

Lawrence MS, Stojanov P, Polak P, Kryukov GV, Cibulskis K, Sivachenko A, Carter SL, Stewart C, Mermel CH, Roberts SA, et al. 2013. Mutational heterogeneity in cancer and the search for new cancer-associated genes. Nature 499: 214-218.

Le DT, Uram JN, Wang H, Bartlett BR, Kemberling H, Eyring $\mathrm{AD}$, Skora $\mathrm{AD}$, Luber $\mathrm{BS}$, Azad NS, Laheru $\mathrm{D}$, et al 2015. PD-1 Blockade in tumors with mismatch-repair deficiency. N Engl J Med 372: 2509-2520.

Ling S, Hu Z, Yang Z, Yang F, Li Y, Lin P, Chen K, Dong L, Cao L, Tao Y, et al. 2015. Extremely high genetic diversity in a single tumor points to prevalence of non-Darwinian cell evolution. Proc Natl Acad Sci 112: E6496-E6505.

Lynch M, Bürger R, Butcher D, Gabriel W. 1993. The mutational meltdown in asexual populations. J Heredity 84 : 339-344.

Maley CC. 2007. Multistage carcinogenesis in Barrett's esophagus. Cancer Lett 245: 22-32.

Maley CC, Galipeau PC, Finley JC, Wongsurawat VJ, Li X, Sanchez CA, Paulson TG, Blount PL, Risques RA, Rabinovitch PS, et al. 2006. Genetic clonal diversity predicts progression to esophageal adenocarcinoma. Nat Genet 38: $468-473$.

Martens EA, Kostadinov R, Maley CC, Hallatschek O. 2011. Spatial structure increases the waiting time for cancer. New J Phys 13.

Martincorena I, Jones PH, Campbell PJ. 2016. Constrained positive selection on cancer mutations in normal skin. Proc Natl Acad Sci 113: E1128-1129.

Marusyk A, Tabassum DP, Altrock PM, Almendro V, Michor F, Polyak K. 2014. Non-cell-autonomous driving of tumour growth supports sub-clonal heterogeneity. Nature 514: 54-58.

McFarland CD, Korolev KS, Kryukov GV, Sunyaev SR, Mirny LA. 2013. Impact of deleterious passenger mutations on cancer progression. Proc Natl Acad Sci 110: $2910-2915$.

McGranahan N, Furness AJ, Rosenthal R, Ramskov S, Lyngaa R, Saini SK, Jamal-Hanjani M, Wilson GA, Birkbak NJ, Hiley CT, et al. 2016. Clonal neoantigens elicit $\mathrm{T}$ cell immunoreactivity and sensitivity to immune checkpoint blockade. Science 351: 1463-1469.

McPherson A, Roth A, Laks E, Masud T, Bashashati A, Zhang AW, Ha G, Biele J, Yap D, Wan A, et al. 2016. Divergent modes of clonal spread and intraperitoneal mixing in high-grade serous ovarian cancer. Nat Genet 48: $758-767$.

Mroz EA, Rocco JW. 2013. MATH, a novel measure of intratumor genetic heterogeneity, is high in poor-outcome classes of head neck squamous cell carcinoma. Oral Oncol 49: $211-215$

Mroz EA, Tward AD, Pickering CR, Myers JN, Ferris RL, Rocco JW. 2013. High intratumor genetic heterogeneity is related to worse outcome in patients with head and neck squamous cell carcinoma. Cancer 119: 3034-3042.

Nakatsuru S, Yanagisawa A, Ichii S, Tahara E, Kato Y, Nakamura Y, Horii A. 1992. Somatic mutation of the $A P C$ gene in gastric cancer: Frequent mutations in very well differentiated adenocarcinoma and signet-ring cell carcinoma. Hum Mol Genet 1: 559-563.

Ng SW, Mitchell A, Kennedy JA, Chen WC, McLeod J, Ibrahimova N, Arruda A, Popescu A, Gupta V, Schimmer AD, et al. 2016. A 17-gene stemness score for rapid determination of risk in acute leukaemia. Nature 540: 433-437.

Nik-Zainal S, Van Loo P, Wedge DC, Alexandrov LB, Greenman CD, Lau KW, Raine K, Jones D, Marshall J, Ramakrishna M, et al. 2012. The life history of 21 breast cancers. Cell 149: 994-1007.

Noble R, Kaltz O, Hochberg ME. 2015. Peto's paradox and human cancers. Philos Trans R Soc Lond B Biol Sci 370: 20150104.

Nordling CO. 1953. A new theory on cancer-inducing mechanism. Br J Cancer 7: 68-72.

Notta F, Chan-Seng-Yue M, Lemire M, Li Y, Wilson GW, Connor AA, Denroche RE, Liang SB, Brown AM, Kim JC, et al. 2016. A renewed model of pancreatic cancer evolution based on genomic rearrangement patterns. Nature 538: 378-382.

Nowell PC. 1976. The clonal evolution of tumor cell populations. Science 194: 23-28.

Ortmann CA, Kent DG, Nangalia J, Silber Y, Wedge DC, Grinfeld J, Baxter EJ, Massie CE, Papaemmanuil E, Menon S, et al. 2015. Effect of mutation order on myeloproliferative neoplasms. N Engl J Med 372: 601-612.

Polyak K. 2008. Is breast tumor progression really linear? Clin Cancer Res 14: 339-341.

Rabinovitch PS, Reid BJ, Haggitt RC, Norwood TH, Rubin CE. 1989. Progression to cancer in Barrett's esophagus is associated with genomic instability. Lab Invest 60: 65-71.

Rausch T, Jones DT, Zapatka M, Stütz AM, Zichner T, Weischenfeldt J, Jäger $N$, Remke $M$, Shih D, Northcott PA, et al. 2012. Genome sequencing of pediatric medulloblastoma links catastrophic DNA rearrangements with TP53 mutations. Cell 148: 59-71.

Rozhok AI, Wahl GM, DeGregori J. 2015. A critical examination of the "bad luck" explanation of cancer risk. Cancer Prev Res (Phila) 8: 762-764.

Shlush LI, Zandi S, Mitchell A, Chen WC, Brandwein JM, Gupta V, Kennedy JA, Schimmer AD, Schuh AC, Yee KW, et al. 2014. Identification of pre-leukaemic haematopoietic stem cells in acute leukaemia. Nature 506: 328 333.

Siegmund KD, Marjoram P, Tavaré S, Shibata D. 2011. High DNA methylation pattern intratumoral diversity implies weak selection in many human colorectal cancers. PLoS ONE 6: e21657. 
Sievers CK, Zou LS, Pickhardt PJ, Matkowskyj KA, Albrecht DM, Clipson L, Bacher JW, Pooler BD, Moawad FJ, Cash BD, et al. 2016a. Subclonal diversity arises early even in small colorectal tumours and contributes to differential growth fates. Gut doi: 10.1136/gutjnl-2016-312232.

Sievers CK, Leystra AA, Clipson L, Dove WF, Halberg RB. 2016b. Understanding intratumoral heterogeneity: Lessons from the analysis of at-risk tissue and premalignant lesions in the colon. Cancer Prevention Res 9: 638-641.

Sinicrope FA, Yang ZJ. 2011. Prognostic and predictive impact of DNA mismatch repair in the management of colorectal cancer. Future Oncol 7: 467-474.

Snyder A, Makarov V, Merghoub T, Yuan J, Zaretsky JM, Desrichard A, Walsh LA, Postow MA, Wong P, Ho TS, et al. 2014. Genetic basis for clinical response to CTLA-4 blockade in melanoma. $N$ Engl J Med 371: 2189-2199.

Sottoriva A, Barnes CP, Graham TA. 2017. Catch my drift? Making sense of genomic intra-tumour heterogeneity. Biochim Biophys Acta 1867: 95-100.

Sottoriva A, Spiteri I, Piccirillo SG, Touloumis A, Collins VP, Marioni JC, Curtis C, Watts C, Tavaré S. 2013a. Intratumor heterogeneity in human glioblastoma reflects cancer evolutionary dynamics. Proc Natl Acad Sci 110: 4009-4014.

Sottoriva A, Spiteri I, Shibata D, Curtis C, Tavaré S. 2013b. Single-molecule genomic data delineate patient-specific tumor profiles and cancer stem cell organization. Cancer Res 73: 41-49.

Sottoriva A, Kang H, Ma Z, Graham TA, Salomon MP, Zhao J, Marjoram P, Siegmund K, Press MF, Shibata D, et al 2015. A Big Bang model of human colorectal tumor growth. Nat Genet 47: 209-216.

Sprouffske K, Athena Aktipis C, Radich JP, Carroll M, Nedelcu AM, Maley CC. 2013. An evolutionary explanation for the presence of cancer nonstem cells in neoplasms. Evol Appl 6: 92-101.

Stephens PJ, Greenman CD, Fu B, Yang F, Bignell GR, Mudie LJ, Pleasance ED, Lau KW, Beare D, Stebbings LA, et al. 2011. Massive genomic rearrangement acquired in a single catastrophic event during cancer development. Cell 144: $27-40$

Sun R, Hu Z, Sottoriva A, Graham TA, Harpak A, Ma Z, Shibata D, Curtis C. 2017. Between-region genetic divergence reflects the mode and tempo of tumor evolution. Nat Genet doi: 10.1038/ng.3891

Suzuki Y, Ng SB, Chua C, Leow WQ, Chng J, Liu SY, Ramnarayanan K, Gan A, Ho DL, Ten R', et al. 2017. Multiregion ultra-deep sequencing reveals early intermixing and variable levels of intratumoral heterogeneity in colorectal cancer. Mol Oncol 11: 124-139.

Tomasetti C, Vogelstein B. 2015. Cancer etiology. Variation in cancer risk among tissues can be explained by the number of stem cell divisions. Science 347: 78-81.
Big Bang Tumor Growth and Clonal Evolution

Uchi R, Takahashi Y, Niida A, Shimamura T, Hirata H, Sugimachi K, Sawada G, Iwaya T, Kurashige J, Shinden $\mathrm{Y}$, et al. 2016. Integrated multiregional analysis proposing a new model of colorectal cancer evolution. PLoS Genet 12: e1005778.

Vermeulen L, Morrissey E, van der Heijden M, Nicholson AM, Sottoriva A, Buczacki S, Kemp R, Tavaré S, Winton DJ. 2013. Defining stem cell dynamics in models of intestinal tumor initiation. Science 342: 995-998.

Vogelstein B, Kinzler KW. 2015. The path to cancer-Three strikes and you're out. N Engl J Med 373: 1895-1898.

Vogelstein B, Papadopoulos N, Velculescu VE, Zhou S, Diaz LA Jr, Kinzler KW. 2013. Cancer genome landscapes. Science 339: 1546-1558.

Wang Y, Waters J, Leung ML, Unruh A, Roh W, Shi X, Chen K, Scheet P, Vattathil S, Liang H, et al. 2014. Clonal evolution in breast cancer revealed by single nucleus genome sequencing. Nature 512: 155-160.

Weaver JM, Ross-Innes CS, Shannon N, Lynch AG, Forshew T, Barbera M, Murtaza M, Ong CA, Lao-Sirieix P, Dunning MJ, et al. 2014. Ordering of mutations in preinvasive disease stages of esophageal carcinogenesis. Nat Genet 46: 837-843.

Weng Z, Spies N, Zhu SX, Newburger DE, Kashef-Haghighi D, Batzoglou S, Sidow A, West RB. 2015. Cell-lineage heterogeneity and driver mutation recurrence in pre-invasive breast neoplasia. Genome Med 7: 28.

Williams MJ, Werner B, Barnes CP, Graham TA, Sottoriva A. 2016. Identification of neutral tumor evolution across cancer types. Nat Genet 48: 238-244.

Yachida S, Jones S, Bozic I, Antal T, Leary R, Fu B, Kamiyama M, Hruban RH, Eshleman JR, Nowak MA, et al. 2010. Distant metastasis occurs late during the genetic evolution of pancreatic cancer. Nature 467: 1114-1117.

Yates LR, Campbell PJ. 2012. Evolution of the cancer genome. Nat Rev Genet 13: 795-806.

Yates LR, Gerstung M, Knappskog S, Desmedt C, Gundem G, Van Loo P, Aas T, Alexandrov LB, Larsimont D, Davies $\mathrm{H}$, et al. 2015. Subclonal diversification of primary breast cancer revealed by multiregion sequencing. Nat Med 21: 751-759.

Zhang J, Fujimoto J, Zhang J, Wedge DC, Song X, Zhang J, Seth S, Chow CW, Cao Y, Gumbs C, et al. 2014. Intratumor heterogeneity in localized lung adenocarcinomas delineated by multiregion sequencing. Science 346: $256-$ 259.

Zhang CZ, Spektor A, Cornils H, Francis JM, Jackson EK, Liu S, Meyerson M, Pellman D. 2015. Chromothripsis from DNA damage in micronuclei. Nature 522: 179-184

Zhu L, Finkelstein D, Gao C, Shi L, Wang Y, López-Terrada D, Wang K, Utley S, Pounds S, Neale G, et al. 2016. Multiorgan mapping of cancer risk. Cell 166: 1132-1146 e7. 


\section{$\&_{\mathrm{CSH}}^{\infty} \&$ Cold Spring Harbor

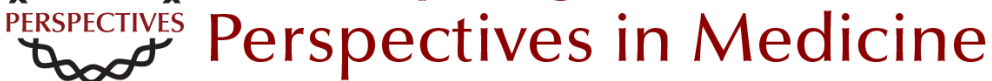

\section{Big Bang Tumor Growth and Clonal Evolution}

Ruping Sun, Zheng Hu and Christina Curtis

Cold Spring Harb Perspect Med 2018; doi: 10.1101/cshperspect.a028381 originally published online July 14,2017

\section{Subject Collection Cancer Evolution}

The Evolution and Ecology of Resistance in Cancer Therapy

Robert A. Gatenby and Joel S. Brown

Big Bang Tumor Growth and Clonal Evolution Ruping Sun, Zheng Hu and Christina Curtis

Observing Clonal Dynamics across

Spatiotemporal Axes: A Prelude to Quantitative

Fitness Models for Cancer

Andrew W. McPherson, Fong Chun Chan and

Sohrab P. Shah

Evolution of Premalignant Disease

Kit Curtius, Nicholas A. Wright and Trevor A. Graham

The Role of Aneuploidy in Cancer Evolution Laurent Sansregret and Charles Swanton

Treatment-Induced Mutagenesis and Selective Pressures Sculpt Cancer Evolution

Subramanian Venkatesan, Charles Swanton, Barry S. Taylor, et al.

Chromosomal Instability as a Driver of Tumor Heterogeneity and Evolution

Samuel F. Bakhoum and Dan Avi Landau

Coevolution of Leukemia and Host Immune Cells

in Chronic Lymphocytic Leukemia

Noelia Purroy and Catherine J. Wu
The Evolution and Ecology of Resistance in

Cancer Therapy

Robert Gatenby and Joel Brown

Phylogenetic Quantification of Intratumor

Heterogeneity

Thomas B.K. Watkins and Roland F. Schwarz

The "Achilles' Heel" of Cancer and Its Implications for the Development of Novel Immunotherapeutic Strategies

Kroopa Joshi, Benjamin M. Chain, Karl S. Peggs, et al.

Homeostasis Back and Forth: An Ecoevolutionary

Perspective of Cancer David Basanta and Alexander R.A. Anderson

Principles of Reconstructing the Subclonal

Architecture of Cancers

Stefan C. Dentro, David C. Wedge and Peter Van LOO

Tumor Microenvironment and Differential

Responses to Therapy

Eishu Hirata and Erik Sahai

Order Matters: The Order of Somatic Mutations Influences Cancer Evolution

David G. Kent and Anthony R. Green

The Cellular Origin and Evolution of Breast

Cancer

Mei Zhang, Adrian V. Lee and Jeffrey M. Rosen

For additional articles in this collection, see http://perspectivesinmedicine.cshlp.org/cgi/collection/ 